\title{
Signing provision in Connected TV: HBB4ALL project
}

\author{
Carlos Alberto Martín
}

\author{
Pilar Orero, José Manuel Menéndez. \\ Guillermo Cisneros
}

\begin{abstract}
This paper explains the work that the HBB4ALL project is carrying out to deploy signing services based on HbbTV (Hybrid Broadcast Broadband TV). HbbTV is an open standard specification for the new Connected TV technology, enabling interoperability of broadcast and broadband contents on the TV set. HBB4ALL proposes to take advantage of this broadcast-broadband convergence for the deployment of access services, including signing, due to the restrictions that this service has traditionally suffered.
\end{abstract}

Index Terms-DTV and broadband multimedia systems; Internet TV; VoD; Interactivity; Field trials; service deployments; Future technologies and services of broadcasting; subjective evaluation techniques; content adaptation and scaling; next generation of broadcasting system

\section{INTRODUCTION}

$\mathrm{C}$ Convergence has been one of the main tendencies in information and communication technologies during the last decades. These convergence processes include services, networks and terminals. Inside this paradigm, a new technology has arisen: Connected TV, as a way to get an effective cooperation between two initially different technologies: broadcast and multimedia broadband systems. Connected TV is characterized by hybrid TV terminals, which are able to receive and to play multimedia content coming from a broadcast network (e.g., the digital terrestrial television or the satellite) and a broadband network (i.e., Internet).

The availability of Internet connection enables new opportunities and services, such as multimedia content personalization on the TV screen. Moreover, it implies the existence of a broadband connection in the household, which also acts like a return channel. In this way, Connected TV is an intrinsically interactive technology.

Connected TV brings as well new opportunities for the deployment of access services for people with disabilities [1]. The existence of the broadband Internet connection can enable the delivery of such services in a personalized, synchronized way, without spending broadcast bandwidth. This is one of the main theses of the HBB4ALL project [2], where authors are collaborating. This papers summarizes the case of sign language interpretation for deaf people, whose practical deployment has traditionally implied some problems [3].

\section{HBBTV AND HBB4ALL}

HBB4ALL is a project co-funded by the European Commission by means of CIP (Competitiveness and Innovation Framework Programme), grant number 621014. HBB4ALL uses an instrument named "pilot", what means that it uses known technologies to deploy new services in almost actual market conditions, as a first step for a future commercial use. Actually, HBB4ALL consists of four different but inter-related pilots:

- Pilot A. Multi-platform subtitle services

- Pilot B. Alternative audio production and distribution

- Pilot C. Automatic user interface adaptation and accessible Connected TV applications

- Pilot D. Sign language interpretation

The planning of these pilots consists of the same four phases, whose core is the "operational phase", starting in summer 2015.

HBB4ALL is based on the HbbTV (Hybrid Broadcast Broadband TV) standard [4]. In fact, HBB4ALL means "Hybrid Broadcast Broadband For All", since its main objective is the extension of Connected TV advantages to all the citizens, including those who have a disability. One key aspect of HBB4ALL is the performance of user tests, including both lab tests and the large scale pilots. In this way, user feedback will allow to know the optimal parameters for quality access service implementations and to ensure service sustainability in the future.

HbbTV [4] is an open specification that enables the 
interoperability between hybrid terminals, multimedia content, service providers and broadband and broadcast networks. For this reason, HbbTV is a valid option even for horizontal markets, where multiple content providers are offering their programmes. Moreover, HbbTV is widely integrated in conventional TV screens in the distribution chain. This open model is preferred by broadcasters, since they have the opportunity to exploit their contents via the broadband network and hybrid terminals. The other Connected TV model consists in proprietary specifications proposed by particular screen manufacturers. In this alternative model, manufacturers create their own broadband portals, which are uniquely visited by the respective screens. In this way, this model, named sometimes "walled garden", do not require standardized and interoperable specifications. "Walled gardens" keep the control under the manufacturers, who sign agreements with content providers to feed their broadband portals.

HBB4ALL is focused on horizontal markets and on making content accessible for as many citizens as possible. For this reason, a standardized, open, and compatible technology like HbbTV is more attractive.

The currently deployed version of HbbTV is 1.5 [4] whereas HbbTV 2.0 specification has been recently released. Different HbbTV releases support different mandatory and optional functionalities.

From its first version, HbbTV has been based on existing and known standardised technologies with the purpose of speeding up the HbbTV application deployment. HbbTV is built on norms from four organizations:

- Open IPTV Forum (OIPF), including the Declarative Application Environment (DAE) and the media formats. The DAE integrates the JavaScript APIs needed in an audio-visual environment to carry out functions on the TV set.

- CE-HTML, i.e., HTML specification for consumer electronics devices, according to CEA-2014 [5].

- DVB (Digital Video Broadcasting, the European organization that creates the digital TV specifications used worldwide) and particularly the norm for transport and signalling of hybrid applications.

- W3C (World Wide Web consortium), including elements such as CSS style sheets, XHTML and the DOM (Document Object Model). DOM is a specification to dynamically access and update the content, structure and style of documents and it allows the TV set to handle the key events, i.e. the user interactions by means of the remote control.

HbbTV references CE-HTML [5] to specify the languages for the applications: XHTML, CSS (the well-known language for style sheets) and JavaScript. HbbTV also includes AJAX (Asynchronous JavaScript and XML), which is a set of web technologies that allows the browser to communicate with the server in background meanwhile web content is depicted.

In other words, HbbTV applications are CE-HTML documents that are interpreted and depicted by a web browser embedded on the TV screen. Since HbbTV is based on web technologies, existing experience in web development can be easily exploited.

Recently, the HbbTV working group has published HbbTV 2.0 specification. This new release integrates ultimate web technologies (such as HTML5) and recent audiovisual consumption paradigms (such as interaction between the TV set and a second screen).

\section{SIGNING PROVISION IN HBB4ALL PROJECT}

HbbTV implies relevant advantages for the deployment of new multimedia contents and services in the current broadcast-broadband convergence process, such as personalization, interactivity and additional content availability. HBB4ALL proposes to take advantage of these functionalities to deploy access services for all the citizens. This section explains the activities in the HBB4ALL project in the specific field of signing interpretation.

Traditionally signing has been provided in an open implementation, i.e. users could not choose the depiction of the service because it is embedded in the programme video and it is visible for all the users. People that do not require this service dislike this kind of implementations since the signing window hides a part of the screen or they find the interpreter distracting. For this reason, programmes with added sign language interpretation are often scheduled in the middle of the night [6]. Moreover, the occlusion on the original picture due to the signing window could cause problems of intellectual property rights [6].

In figure 1, a traditional implementation of signing is shown. The signer appears in a small window on the main video signal. This snapshot is a sample of signing service of RTP, one of the HBB4ALL partners.

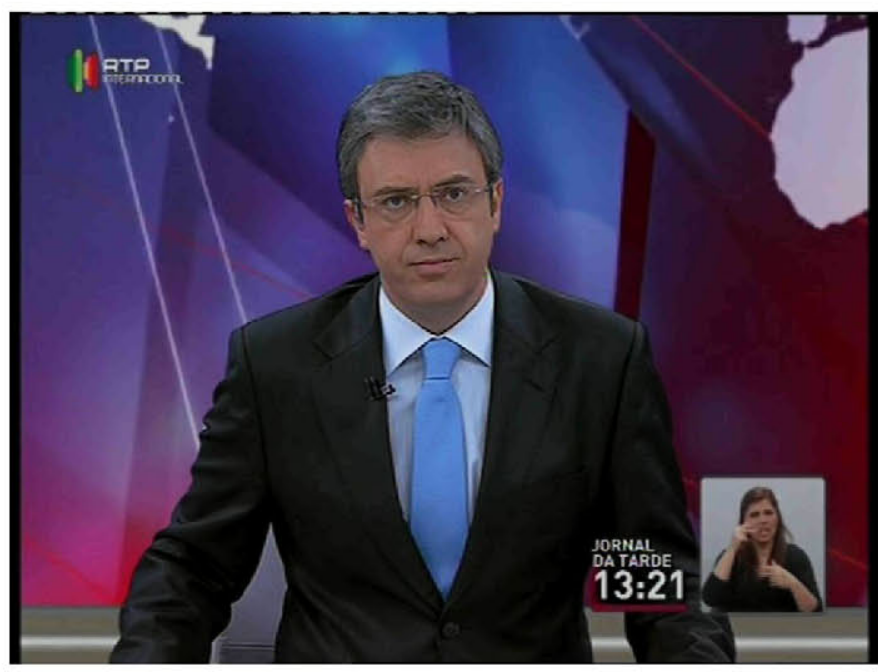

Fig. 1. Common implementation of signing service in broadcast programmes. This snapshot comes from RTP, the national Portuguese broadcaster.

Due to the abovementioned problems and restrictions, a closed (selective) component for the signing service is a strong need for digital TV channels, as said by the European Broadcasting Union (EBU) in technical reports on access services [6]. HBB4ALL proposes solutions for the deployment 
of closed (selective) implementation of signing, taking advantage of the broadband connection and broadband multimedia system capabilities.

As explained in this section, HBB4ALL is working on three different technical developments to provide the signing service:

- An HbbTV application to deliver the signing service via Internet and the broadband multimedia features of the hybrid terminals.

- A web-based approach to provide a fully customisable signing service by means of a double-screen for PC and other connected devices.

- A signing service based on an avatar (virtual interpreter) for a concrete semantic domain (weather forecast).

These technical deployments are detailed in the next subsections.

\section{A. HbbTV signing application}

This signing implementation is led in HBB4ALL by RBB, the public broadcaster of the regions of Berlin and Brandenburg in Germany.

This signing application takes advantage of the HbbTV features to provide a closed implementation of the signing service. A truly hybrid approach would involve the delivery of the programme via the broadcast network (e.g., digital terrestrial television or satellite), the delivery of the signing interpretation video via the broadband network and a hybrid terminal able to mix both video signals in a picture-in-picture function. This implementation would be very valuable since it would enable directly customisation options (position and size of the signing windows in the screen). However, it would require a double-decoder TV terminal and this is an optional feature in HbbTV specifications.

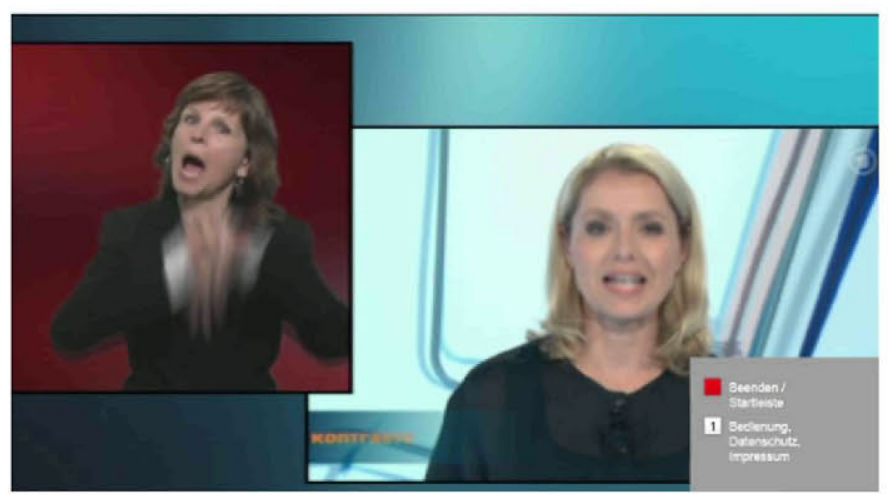

Fig. 2. Preliminary implementation of the HbbTV signing application developed by RBB in HBB4ALL

For this reason, the project has chosen a different approach: the provision of a pre-mixed picture-in-picture signing service via the broadband network. This approach enables a closed implementation: if a viewer wants to watch the signing service for a concrete broadcast programme, she or he can enable it in the HbbTV launcher (the launcher or red-button application is a graphical interface that links the rest of applications provided by the broadcaster) and from that moment the broadband network is used to provide a video stream that integrates both signals: programme and signing implementation. Figure 2 shows a snapshot of the preliminary RBB implementation of this service.

This technical solution takes advantages of HbbTV features such as multimedia format support and real streaming capabilities (instead of file progressive download).

\section{B. Web-based signing service}

Inside the mentioned paradigm of broadband and broadcast convergence, RTP and UPM, HBB4ALL partners, are also working in a different approach to provide the signing service. RTP is currently supplying this access service by means of a small window in the broadcast programmes as shown in figure 1. Additionally, to improve content accessibility, RTP offers the signing service in a larger window for live programmes in its website. This feature is named "double screen" since, as shown in figure 3 , two equal screens are provided: on the one hand, the programme signal (as it is being broadcasted simultaneously, including the small window for signing) and on the other hand, the signing service.

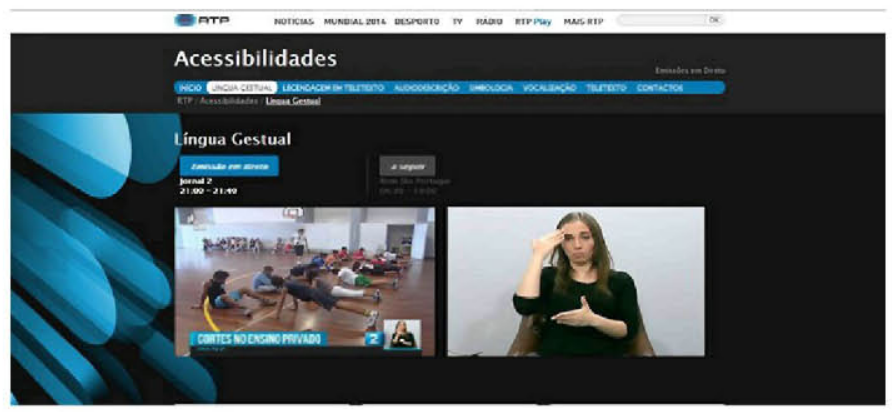

Fig. 3. Snapshot of a PC browser showing the double-screen functionality for signing in RTP website.

Since this service is just provided for live content at this point in time, HBB4ALL proposes to extend it for catch-up content, enabling a fully customisable implementation. The pilot deployment requires changes in the production workflow to record the additional signal of the interpreter and to make it available in a web page. The content for the pilot, starting in summer 2015, will not be available in the RTP website but in an alternative web server.

Moreover, RTP usually collaborates with Portuguese associations of people with a hearing impairment. RTP counts on these associations to participate in the pilot validation.

\section{Avatar signing application}

One of the main contributions of Vicomtech-IK4 to the HBB4ALL project is being an avatar signing service. It is not focused on a pilot deployment but on the validation of the technology and user tests. It is able to translate Spanish (spoken language) into Spanish Sign Language (known as 
LSE) by means of an avatar or virtual interpreter.

This application includes several technological modules, such as a text-to-sign language translation module, a gesture capture system to create an internal sign corpus, a sign dictionary, an animation engine and a rendering module. Every semantic domain requires its specific sign dictionary. In HBB4ALL, the validation of this technology is focused on weather forecast as specific semantic domain. Figure 4 shows a snapshot of a video generated in the project.

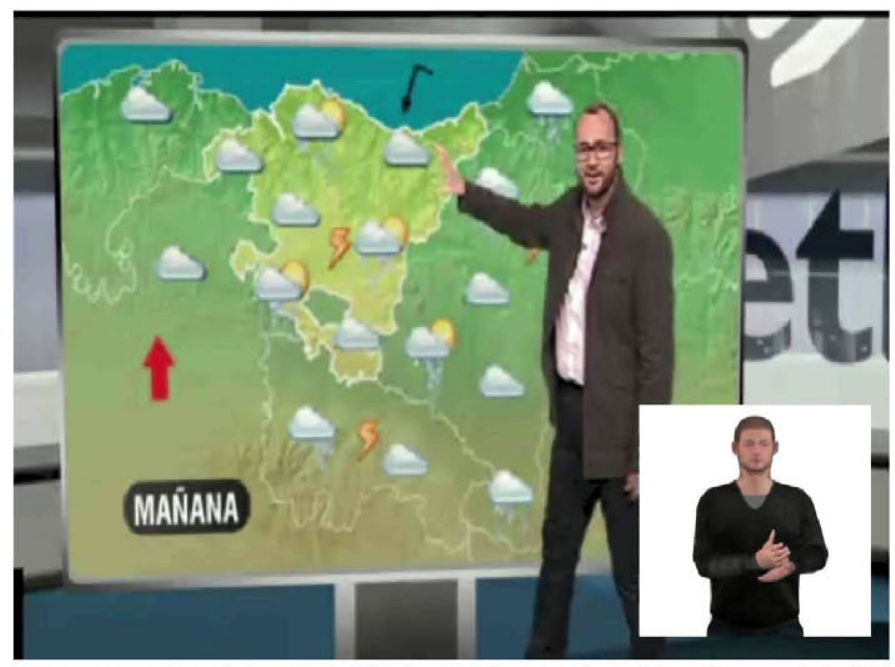

Fig. 4. Snapshot of the avatar signing service developed by Vicomtech inside the HBB4ALL project.

Once the performance of this application is validated, it could be easily adapted to other semantic domains in further developments after generating the suitable sign dictionary.

\section{USER TESTS}

Not only plans HBB4ALL the deployment of large scale pilots in almost real operation conditions. User and lab tests are a key point in the case of sign language and the rest of HBB4ALL pilots. The objective is to acquire new knowledge about the optimal parameters for the signing service implementation. This quality requirement is also fulfilled by the customisation features, which are present in the project implementations to achieve satisfying services for the users.

Tests for the service were performed at two levels: user and lab tests. Test complexity and length were an issue hence it was considered important to narrow down the number of features and variables to be assessed experimentally. Different research methods (both qualitative and quantitative) were designed previous to the actual lab tests and pilots. The tests include: eye-tracking measurement, visual memory, and linguistic comprehension. There was also a preference survey to assess the end-user experience after watching a 3-minute clip for each condition.

Quantitative data gathering tests were performed collecting one hundred screen shots from broadcasters throughout 42 countries. They were retrieved via the online platform Sign Language Television for the Deaf (http://signlangtv.org/) to give us a full perspective of what formal features could be attested on TV within and outside EU. These data showed a great deal of variation. Qualitative data collection aimed to gather information from the two groups directly involved in sign language on TV. On the one hand, sign language interpreting professionals that currently work or have worked on TV and, on the other, signing deaf people who are the targeted audience. For each of these groups a different qualitative data collection method was designed and developed: interviews with the interpreters and focus groups with signing deaf users. After the qualitative data collection it was agreed that the first features to be tested in lab conditions would be size and position of the sign language interpreter onscreen. To avoid lab tests longer than one hour, it was also established that for each of the formal features only two variables would be measured. The variables for the sub-screen position were right and left positions, which were the ones mostly discussed by the users in the focus groups. In the case of the interpreter sub-screen size, more variation had been attested in the data and it had been classified in three sizes: small, medium and big (roughly $1 / 8,1 / 3$ and $1 / 2$ screen). In order to choose two of these sizes, a pretest with signing deaf users with three-minute news clips showing the different size variables was designed: the small and medium size will be tested in lab conditions. The compilation samples performed in the project has revealed the many possible implementations in terms of interpreter position and size, main video size, shot size, use of chroma-key, gender of the interpreter, background colour, clothing colour, among other features. These parameters directly affect sign language on-screen readability and thus have a direct impact on accessibility for deaf users whose first linguistic option is a sign language.

\section{PRELIMINARY CONCLUSIONS}

Despite of the operational phase of the pilots in HBB4ALL has not started yet, some preliminary conclusions can be already considered. First, the HbbTV specification and the HBB4ALL project are good examples of the interoperability between broadband and broadcast multimedia systems to provide innovative services and contents. Second, although HbbTV was not specifically designed to provide access services, it includes a variety of functionalities that can be used for this purpose. And finally, in a general manner, the history of the broadcast technology shows how new specifications, paradigms and services (e.g., Connected TV, interactive TV, teletext, multichannel audio, etc.) are also available to provide innovative services for disadvantaged groups of citizens, e.g. people with disabilities. The operational phase of HBB4ALL will be useful to extract information about the access service performance in real exploitation conditions and to get feedback from users.

\section{REFERENCES}

[1] C. A. Martín, J. M. Menéndez and G. Cisneros, "Connected TV: new opportunities for the accessibility". NEM SUMMIT 2012. Istambul. October 2012.

[2] http://www.hbb4all.eu 
[3] Frans de Jong. "Access Services for digital television". EBU Technical Review. October 2004. Available https://tech.ebu.ch/docs/techreview/trev_300-de_jong.pdf

[4] European Telecommunications Standards Institute (ETSI). "Hybrid Broadcast Broadband TV". ETSI TS 102796 V1.2.1. Available in: http://www.etsi.org/deliver/etsi ts $\% 5$ C102700_102799\%5C102796\%5C 01.02.01_60\%5Cts_102796v010201p.pdf
[5] Consumer Electronic Association. CEA2014 "Web-based Protocol and Framework for Remote User Interface on UPnPTM Networks and the Internet (Web4CE)". Jul, 2007.

[6] European Broadcasting Union (EBU). "EBU Report on Access Services -includes recommendations". EBU Technical - Information I44-2004. June 2004. Available in: https://tech.ebu.ch/docs/i/i044.pdf 